\title{
IX. Measurement of the expansibility of a hard Jena glass
}

\author{
J.T. Bottomley M.A. D.Sc. F.R.S. \& W.T. Evans
}

To cite this article: J.T. Bottomley M.A. D.Sc. F.R.S. \& W.T. Evans (1901) IX. Measurement of the expansibility of a hard Jena glass, Philosophical Magazine Series 6, 1:1, 125-128, DOI: $10.1080 / 14786440109462596$

To link to this article: http://dx.doi.org/10.1080/14786440109462596

曲 Published online: 08 Jun 2010.

Submit your article to this journal $[\pi$

Џ Article views: 3

Q View related articles $\asymp$ 
IX. Measurement of the Expansibility of a Hard Jena Glass. $B y$ J. T. Botтomley, M.A., D.SC., F.R.S., and W.'T. Evals *.

I $\mathrm{N}$ the course of investigations which we are carrying out on the subject of Thermal Radiation it became necessary to construct an Air-Thermometer which should be capable of being used at high temperatures, above the softeningpoint of ordinary English glass. The form of air-thermometer employed is that which was described in the Philosophical Magazine for $1888+$ by one of the present writers. In this thermometer, which is a constant-volume air-thermometer, the volume-gauge and pressure-gauge are separated, and only connected by means of indiarubber pressure-tubing. The instrument has proved eminently satisfactory, and convenient to work with.

The volume-gauge is the only part which it is necessary to make of hard glass, and, after some trials with specimens of the Jena glass of Messrs. Schott \& Genossen, who specify three kinds of hard glass, we determined to use the glass termed by them "Verbrennungsröhrenglas." This is a very hard glass, little acted on by water or alkalis (though this property was not of much consequence for our purpose). It is slightly green in colour, and slightly opalescent. It is just possible to work it with an ordinary blowpipe, but for any complicated construction an oxygen flame is required.

In order to use this glass for the volume-gauge of the airthermometer, it was necessary to determine the expansibility of the glass. This we have done; and the present note is published in the hope that the results may be of use to others. We were surprised to find so small an amount of expansibility. This property makes the glass of special value for thermometric purposes, at high temperatures.

To determine the expansibility of the glass, the weightthermometer method was used, and the cubical expansion was found directly. This seemed mare satisfactory than the determination of the linear expansion, and the inference, therefrom, of the cubical expansion.

The bulb of the air-thermometer is a tube of Jena glass $12 \mathrm{~cm}$. long and $1.5 \mathrm{~cm}$. in diameter inside. A similar tube

* Communicated by Lord Kelvin.

† J. T. Bottomley, "On a Practical Constant-Volume Air-Thermometer," Proc. Roy. Soc. Edin. Dec. 19th, 1887, and Phil. Mag. August 1888. 
was taken and fitted with a T-tube of narrow gauge, as shown in the figure, and a reservoir $F$, to aid in the process of filling the bulb $\mathrm{E}$. This apparatus was carefully weighed, and it was then filled with pure mercury; an extra quantity of mercury being left in the reservoir $\mathrm{F}$.

To effect the filling, the end $\mathrm{C}$ was closed with a stopper, and the bulb $\mathrm{F}$ partially filled with mercury. A waterpump was then applied at $B$, and a quantity of air was taken out of $\mathrm{E}$, which bubbled up through the mercury in the reservoir $F$. The air was now allowed to flow back through $B$, and the mercury in $F$ was driven into the bulb E. By repetitions of this process the bulb E was filled, except for a small bubble, which was used to roll over the inside of the glass, and remove adhering air-bubbles between the mercury and the glass. In the end there was no air left, except a minute bubble at $D$, but there was still an excess of mercury remaining in the reservoir $\mathbf{F}$.

To get rid of the bubble at $\mathrm{D}$, the bulb $\mathrm{E}$ was cooled under the watertap; and in this way a little more mercury passed back into $\mathrm{E}$, without disturbing the bubble at D. By now warming the bulb $\mathrm{E}$ with the hand, the last small bubble passed out, and $\mathbf{a}$

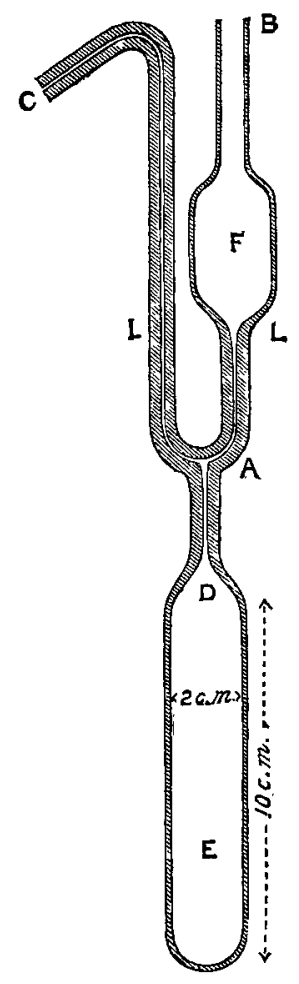
perfectly continuous volume of mercury was obtained. The stopper at $C$ was removed to $B$, and a drying-tube connected at $\mathrm{C}$, to prevent condensation of moisture from the air when the bulb was put into ice.

The bulb and tube, up to the level LL, were then placed in melting ice, and kept there till there could be no doubt that the whole was at ice-cold temperature. This being done, the excess of mercury, down to the point $A$, was removed by the application of the water-pump to $B$ (after removing the stopper), which drew a current of dried air through the tube $\mathrm{B} \mathrm{FAC}$, and cleared out the excess of mercury above the point A. This method was found to act perfectly.

The tube, with its mercury, was now very carefully dried and weighed. 
It was next transferred to a couper steam-jacket of the ordinary kind used for fixing the boiling-points of thermometers, and raised to the boiling-point of water ; the ordinary precautions being taken, and the barometer read in order to apply the correction for reduction to Steam Temperature, at Standard Pressure.

During this operation a quantity of mercury was driven up into the tube FAC, and this was removed, as before, by an application of the vacuum of the water-pump. The tube was then allowed to cool, and was dried and weighed.

The whole process was extremely easy; and the only important source of error was that due to the capillarity of the mercury at the top; which, with clean glass and clean mercury, and with so large a total quantity of mercury, was very small. Spezial care was taken to make the volume of the joint at $A$ as small as possible, in order to minimise this source of error. The operations are much easier than those involved in the handling and weighing of a weight-thermometer of the ordinary kind at the ice-temperature.

The following exainples show the numerical results of one of the experiments :-

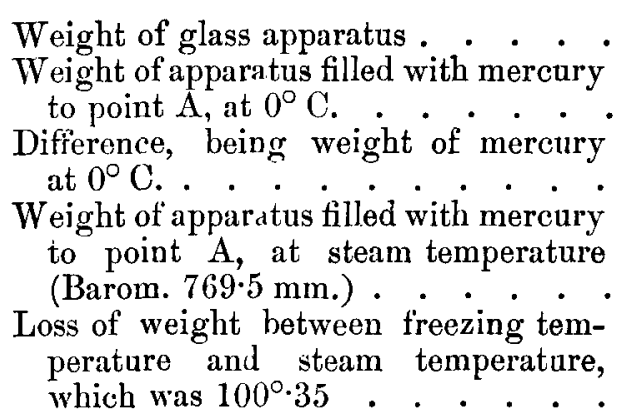

Frammes.

$83 \cdot 880$

$539 \cdot 665$

Hence the apparent expansion of mercury in this glass, between $0^{\circ}$ and $100^{\circ}$, was

$$
\frac{7 \cdot 615 \times 100}{463.40 \times 100 \cdot 35}=0 \cdot 01633
$$

Subtracting this from the known expansion of mercury between $0^{\circ}$ and $100^{\circ}$, viz. 0.01815 , we obtain for the cubic expansion of the glass 0.00177 .

Other numbers obtained in a similar way were as follow :-

(2) . . . . . . 0.0018z.

(3) . . . . . 0.00183 . 
For another specimen of the same glass we obtained 0.00181.

A similar experiment was made with Jena "Verbundglas" D.R.P. No. 61573 , by removing the bulb E, and joining on a bulb of the D.R.P. glass.

The result of a single experiment showed its cubical expansibility to be 0.0022 between $0^{\circ}$ and $100^{\circ}$; a result not very different from that iof ordinary German glass.

\section{On the Viscosities of Mixtures of Liquids and of} Solutions. By Charles H. Lees, D.Sc.*

T $N$ recent years many determinations of the riscosities of 1 mixtures of liquids and of solutions have been made in the endeavour to discover the law of connexion of the viscosity of a mixture with the relative amounts and viscosities of its constituents. As a result several empirical formulæ have been proposed which represent the observations with more or less accuracy. Of these formulx, the one first used by Arrhenius in 1887, and since then by Reyer, Wagner, Lauenstein, and Kanitz, for weak salt-solutions or for mixtures of liquids when one liquid is present to the extent of 90 per cent. or more, seems to be the most valuable. According to it the viscosity $\eta$ at a temperature $t$, of a mixture which contains in 1 c.c., $v_{1}$ c.c. of a liquid of viscosity $\eta_{1}, v_{2}$ c.c. of one of viscosity $\eta_{2}$, \&c., at temperature $t$, is given by the equation

or

$$
\eta=\eta_{1}^{v_{1}} \cdot \eta_{2}^{v_{2}} \cdot \eta_{3}^{v_{3}} \ldots \ldots
$$

$$
\log \eta=v_{1} \log \eta_{1}+v_{2} \log \eta_{2}+v_{3} \log \eta_{3}+\ldots \ldots
$$

so long as one constituent is present to the extent of 90 per cent. or more. It is the object of the present paper to consider whether this formula can be supported on theoretical grounds, to see how far it agrees with the observed facts for mixtures generally, and if the agreement is unsatisfactory to replace it by a more suitable expression.

To arrive at a theory for mixtures of fluids which have no chemical action on each other, we may suppose the volume of a mixture to be divided into elementary volumes by, say, three series of parallel planes. Each of these elementary volumes we may suppose occupied exclusirely by one constituent. The limit of the calculated viscosity of a medium so built up when the elementary parts are taken indefinitely small, may be taken as the viscosity of the mixture.

* Communicated by the Physical Society.: read June 8, 1900. 